\title{
Zagreb Connection Index of Drugs Related Chemical Structures
}

\author{
Muhammad Imran Qureshi ${ }^{1(\mathbb{C})}$, Asfand Fahad ${ }^{1, *(\mathbb{C})}$, Muhammad Kamran Jamil ${ }^{2(\mathbb{C})}$, Sohail Ahmad ${ }^{1(\mathbb{D})}$ \\ 1 Department of Mathematics, COMSATS University Islamabad, Vehari 61100, Pakistan; imranqureshi18@gmail.com \\ (M.I.Q.); asfandfahad1@yahoo.com (A.F.); sohailahmadmirza@yahoo.com (S.A.) \\ 2 Department of Mathematics, Riphah Institute of Computing and Applied Sciences (RICAS), Riphah International \\ University, 14 Ali Road, Lahore, Pakistan; m.kamran.sms@gmail.com (M.K.J.) \\ * Correspondence: asfandfahad1@yahoo.com;
}

Scopus Author ID 56678506000

Received: 20.11.2020; Revised: 15.12.2020; Accepted: 18.12.2020; Published: 21.12.2020

\begin{abstract}
Topological indices are used to test the medicine and pharmacology characteristics of drugs and their molecular structures. The modified first Zagreb connection number index is defined to be used in the analysis of drug structures. In this paper, by means of drug molecular structure analysis and vertex partitioning method, we compute the modified first Zagreb connection number index of graphene, polyomino chains, and Benzenoid systems, etc. These structures are used widely in molecular drug graphs.
\end{abstract}

Keywords: Zagreb index; modified first Zagreb connection number index; drugs-related chemical structures.

(C) 2020 by the authors. This article is an open-access article distributed under the terms and conditions of the Creative Commons Attribution (CC BY) license (https://creativecommons.org/licenses/by/4.0/).

\section{Introduction}

Chemical compounds can be represented by (molecular) graphs in which vertices correspond to the atoms while edges represent the covalent bonds between atoms [1,2]. Let $\mathrm{G}=(\mathrm{V}(\mathrm{G}) ; \mathrm{E}(\mathrm{G}))$ be a molecular graph with vertex set $\mathrm{V}(\mathrm{G})$ and edge set $\mathrm{E}(\mathrm{G})$. The number of elements in $V(G)$ and $E(G)$ is called the order and the size of the graph $G$. The number of vertices adjacent to the vertex $v \in V(G)$ is called the degree of the vertex $v$, which is denoted as $d(v)$. The length of the shortest path between two vertices $u$ and $v$ is called the distance between these vertices, and it is denoted as $d(u ; v)$. The notation $\tau_{v}$ for the vertex $v$ represents the number of vertices at a distance 2 from the vertex $v, \tau_{v}$ is known as the connection number of $\mathrm{v}$.

As a usual path, cycle and complete graphs with $n$ vertices are denoted by $\mathrm{P}_{\mathrm{n}} ; \mathrm{C}_{\mathrm{n}}$, and $\mathrm{K}_{\mathrm{n}}$, respectively.

A molecular graph's topological index is a numerical quantity, which is a graph invariant under graph isomorphism. In theoretical chemistry, the physiochemical properties of chemical compounds are often modeled by the topological indices.

First and second Zagreb indices of a simple graph $\mathrm{G}$ are defined as:

$$
\begin{aligned}
& M_{1}(G)=\sum_{v \in V(G)} d(v)^{2}=\sum_{u v \in E(G)}(d(u)+d(v)) \\
& M_{2}(G)=\sum_{u v \in E(G)} d(u) d(v)
\end{aligned}
$$


Followed by the first and second Zagreb indices, Furtula et al. [3] introduced the forgotten topological index, which was defined as

$$
F(G)=\sum_{v \in V(G)} d(v)^{3}=\sum_{u v \in E(G)}\left(d(u)^{2}+d(v)^{2}\right.
$$

Numerous work on these indices has been done so far [4-27]. In 1972, Gutman et al. [28] showed that the total $\pi$-electron energy of a molecule depends on the first Zagreb index and the forgotten topological index. In the same paper, the quantity $\sum_{v \in V(G)} d(v) \tau_{v}$ was shown to influence the $\pi$-electron energy, but the topological index was never again investigated and left to oblivion. Trinajstic et al. [29] reintroduced this quantity and named as modified first Zagreb connection index and denote it by $\mathrm{ZC}^{*}$, i.e.

$$
Z C_{1}^{*}(G)=\sum_{v \in V(G)} d(v) \tau_{v}
$$

This paper computed the newly introduced modified first Zagreb connection number index of special molecular graphs discussed in chemicals, nanomaterials, and pharmaceutical engineering.

\section{Materials and Methods}

In theoretical chemistry, the physiochemical properties of chemical compounds are often modeled by the topological indices. In the definition of modified first Zagreb connection

$$
Z C_{1}^{*}(G)=\sum_{v \in V(G)} d(v) \tau_{v}
$$

$d(v)$ is representing the degree of vertex $v$ and $\tau_{v}$ is representing the number of vertices in graph, which are at a distance 2 from $v$. $\tau_{v}$ is called the connection number of $v$.

This article computes the first modified Zagreb connection for the chemical graphs of 2-Dimensional Graphene sheet, 2-Dimensional Zig-zag polyomino chain, and Benzenoid systems, etc. We will use these graphs' vertex sets based on the degrees and connection numbers of the vertices. These partitions are given in the form of tables.

\section{Results and Discussion}

\subsection{Modified first Zagreb connection index of graphene sheet $G(m, n)$.}

Graphene is the main element of certain carbon allotropes, including graphite, fullerenes, and charcoal. Graphene is a planar sheet of carbon atoms that are densely packed in a honeycomb crystal lattice. A two-dimensional graphene sheet $G(m ; n)$, where $m$ is the number of columns and $\mathrm{n}$ is the number of rows, is shown in Fig. 1.

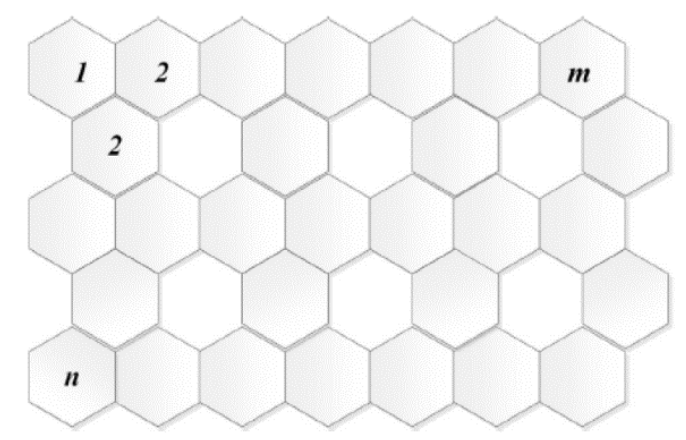

Figure 1. 2-dimensional graphene sheet. 
The molecular graph of graphene sheet $G(m ; n)$ contains $2(m n+m+n)$ number of vertices. There are $2(m+n+1)$ vertices of degree two and $2 m n-2$ vertices are of degree three. Table 1 shows the vertex set's partitions based on the degree and the connection number of the vertices.

Table 1. Vertex set the partition of $\mathrm{G}(\mathrm{m} ; \mathrm{n})$ based on the degree and the connection number of the vertices.

\begin{tabular}{ccc}
\hline $\boldsymbol{d}(\boldsymbol{v})$ & $\boldsymbol{\tau}_{\boldsymbol{v}}$ & Number of vertices \\
\hline $\mathbf{2}$ & 2 & 2 \\
\hline $\mathbf{2}$ & 3 & $2 n+4$ \\
\hline $\mathbf{2}$ & 4 & $2 m-4$ \\
\hline $\mathbf{3}$ & 4 & $2 m$ \\
\hline $\mathbf{3}$ & 5 & $2 n-4$ \\
\hline $\mathbf{3}$ & 6 & $2(m-1)(n-1)$ \\
\hline
\end{tabular}

Theorem 3.1.1. Consider the graph of graphene sheet $\mathrm{G}(\mathrm{m} ; \mathrm{n})$, where $\mathrm{m} ; \mathrm{n}>1$, then the modified first Zagreb connection index of $\mathrm{G}(\mathrm{m} ; \mathrm{n})$ is

$$
Z C_{1}^{*}(G(m, n))=36 m n+4 m+6 n-24 .
$$

Proof. The 2-dimensional structure of graphene sheet $\mathrm{G}(\mathrm{m} ; \mathrm{n})$ has $2(\mathrm{mn}+\mathrm{m}+\mathrm{n})$. Table 1 shows the vertex set's partition is based on the degree and the connection number of the vertices. With the help of Table 1, we can compute the modified first Zagreb connection index of $G(m ; n)$ as follows

$$
\begin{aligned}
& Z C_{1}^{*}(G(m, n))=\sum_{v \in V(G(m, n))} d(v) \tau_{v}=2 \cdot 2 \cdot 2+(2 n+4) \cdot 2 \cdot 3+(2 m-4) \cdot 2 \cdot 4+2 m \cdot 3 \cdot 4 \\
& +(2 n-4) \cdot 3 \cdot 5+2(m-1)(n-1) \cdot 3 \cdot 6
\end{aligned}
$$

By easy calculation, we have

$$
Z C_{1}^{*}(G(m, n))=36 m n+4 m+6 n-24
$$

\subsection{Modified topological index of polyomino chains of $2 n$-cycles.}

A polyomino system is a nite 2-connected plane graph. A regular square of length one surrounds each interior face (cell). In other words, it is an edge-connected union of cells in the planar square lattice. Polyominoes have a long and rich history [30]. A two-dimensional zigzag polyomino chain is shown in Fig. 2.

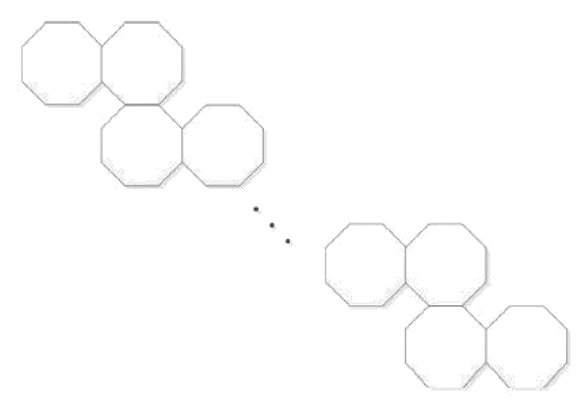

Figure 2. 2-dimensional zig-zag polyomino chain.

From Fig. 2 note that there are $12 n+2$ vertices in this molecular graph. We partitioned the vertex set based on the degree and the connection number of the vertices, and shown in detail in Table 2. 
Table 2. Vertex set the partition of the zig-zag polyomino chain based on the degree and the connection number of the vertices.

\begin{tabular}{ccc}
\hline $\boldsymbol{d}(\boldsymbol{v})$ & $\boldsymbol{\tau}_{\boldsymbol{v}}$ & Number of vertices \\
\hline 2 & 2 & $4 n+4$ \\
\hline 2 & 3 & $4 n$ \\
\hline 3 & 4 & 2 \\
\hline 3 & 5 & $2 n-4$ \\
\hline
\end{tabular}

Theorem 3.2.1. Consider the molecular graph $\left(Z_{n}\right), n \geq 1$, of zig-zag polyomino chains; then its modified first Zagreb connection number equals to

$$
Z C_{1}^{*}\left(Z_{n}\right)=100 n-20
$$

Proof. The molecular graph of the zig-zag polyomino chain $\left(Z_{n}\right)$ contains $12 n+2$ vertices; among them, $8 n+4$ vertices are of degree two, and $4 n 2$ vertices are of degree three. From Table 2 and the definition of the modified first Zagreb connection index, we have

$$
Z C_{1}^{*}\left(Z_{n}\right)=\sum_{v \in V\left(Z_{n}\right)} d(v) \tau_{v}=(4 n+4) \cdot 2 \cdot 2+4 n \cdot 2 \cdot 4+2 \cdot 3 \cdot 4+(4 n-4) \cdot 3 \cdot 5 .
$$

After a simple calculation, we have

$$
Z C_{1}^{*}\left(Z_{n}\right)=100 n-20
$$

A linear polyomino chain is denoted as $\mathrm{L}_{\mathrm{n}}$, and is shown in Fig. 3. This structure has $2(n+1)$ vertices, and four vertices are of degree two, and the other 2(n-1) vertices are of degree 3. The partition of the vertex set based on the degree and the connection number of vertices is shown in Table 3.

\begin{tabular}{|c|c|c|c|}
\hline$d(v)$ & $\boldsymbol{\tau}_{v}$ & \multicolumn{2}{|c|}{ Number of vertices } \\
\hline 2 & 2 & & \\
\hline 3 & 3 & & \\
\hline 3 & 4 & & \\
\hline 1 & 2 & 3 & $\mathrm{n}$ \\
\hline
\end{tabular}

Table 3. Vertex set the partition of linear polyomino chain based on the degree and the connection number of the vertices.

Figure 3. A linear polyomino chain.

Theorem 3.2.2. The modified first Zagreb connection number index of the molecular graph of the linear polyomino chain, $\mathrm{L}_{\mathrm{n}} ; \mathrm{n} \geq 2$, is

$$
Z C_{1}^{*}\left(L_{n}\right)=24 n-8
$$

Proof. The molecular structure of a linear polyomino chain contains $2(n+1)$ vertices. The partitioning of the vertex set of the molecular structure $L_{n}$ is described in Table 3. By definition and Table 3, we have

$$
Z C_{1}^{*}\left(L_{n}\right)=\sum_{v \in V\left(L_{n}\right)} d(v) \tau_{v}=4 \cdot 2 \cdot 2+4 \cdot 3 \cdot 3+2(n-3) \cdot 3 \cdot 4=24 n-20 .
$$

3.3. Modified first Zagreb connection index topological index of polyomino chains of triangular Benzenoid. 
This section computes the modified first Zagreb connection index of the triangular Benzenoid graph shown in Fig. 4. This graph has $n^{2}+4 n+1$ vertices.

Theorem 3.3.1. Let $H_{n}$, where $n \geq 1$, be the molecular graph of the triangular Benzenoid system. Its modified first Zagreb connection number is

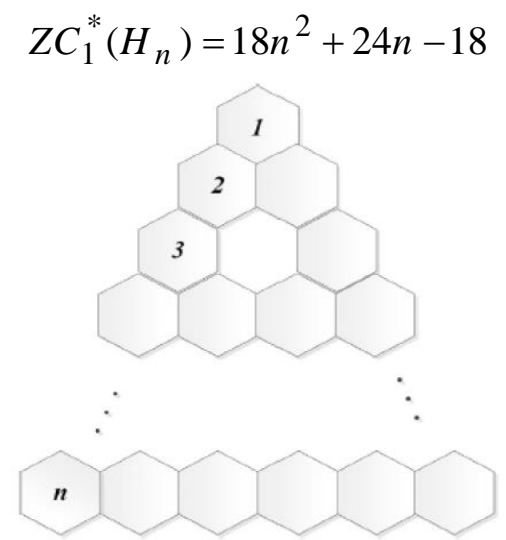

Figure 4. Graph of triangular Benzenoid G[n].

Proof. The molecular graph of the triangular Benzenoid system has $n^{2}+4 n+1$. There are $3(n+1)$ vertices with degree two and $n^{2}+n-2$ vertices with degree three. The vertex set of graph $H$ is partitioned based on the degree and the connection number of the vertices. Table 4 illustrates these details. Using Table 4 we can obtain the result

$$
Z C_{1}^{*}\left(H_{n}\right)=\sum_{v \in V\left(H_{n}\right)} d(v) \tau_{v}=3 \cdot 2 \cdot 2+6 \cdot 2 \cdot 3+(3 n-6) \cdot 2 \cdot 4+3(n-1) \cdot 3 \cdot 4+(n-1)^{2} \cdot 3 \cdot 6=18 n^{2}+24 n-18
$$

Table 4. Vertex set the partition of the triangular Benzenoid system based on the degree and the connection number of the vertices.

\begin{tabular}{ccc}
\hline $\boldsymbol{d}(\boldsymbol{v})$ & $\boldsymbol{\tau}_{\boldsymbol{v}}$ & Number of vertices \\
\hline 2 & 2 & 3 \\
\hline 2 & 3 & 6 \\
\hline 2 & 4 & $3 n-6$ \\
\hline 3 & 4 & $3 n-3$ \\
\hline 3 & 6 & $(n-1)^{2}$ \\
\hline
\end{tabular}

\subsection{Modified first Zagreb connection index of molecular bridge structures.}

Let $\left\{G_{i}\right\}_{i=1}^{t}$ be a set of nite pair-wise disjoint molecular graphs such that $\mathrm{v}_{\mathrm{i}} \in \mathrm{V}(\mathrm{G})$. The bridge molecular graph $\mathrm{B}\left(\mathrm{G}_{1} ; ; \mathrm{G}_{\mathrm{t}}\right)=\mathrm{B}\left(\mathrm{G}_{1}, \ldots, \mathrm{G}_{\mathrm{t}}\right)$ of $\left\{G_{i}\right\}_{i=1}^{t}$ concerning the vertices $\left\{v_{i}\right\}_{i=1}^{t}$ is yielded from the molecular graphs $\mathrm{G}_{1}, \ldots, \mathrm{G}_{\mathrm{t}}$ in which the vertices $\mathrm{v}_{\mathrm{i}}$ and $\mathrm{v}_{\mathrm{i}+1}$ are connected by an edge for $\mathrm{i}=1 ; 2, \ldots, \mathrm{t}-1$. A bridge graph is shown in Fig. 5 . If $\mathrm{G}_{1}=\mathrm{G}_{2}=, \ldots,=\mathrm{G}_{\mathrm{t}}=\mathrm{G}$ with $v \in V\left(G_{i}\right)$ for all $i$, then the notation for bridge graph is $B_{t}(G ; v)=B(G, \ldots, G ; v, \ldots, v)$.

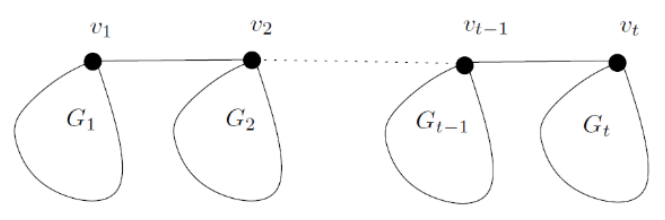

Figure 5. A bridge molecular graph.

This section computes the modified first Zagreb connection index of bridge graphs among paths, cycles, and complete graphs, respectively. 
Theorem 3.4.1. Let $\mathrm{n} ; \mathrm{t}>2$, then

$$
\begin{aligned}
& 1-Z C_{1}^{*}\left(B_{t}\left(P_{n}, v\right)\right)=4 t n+8 t-28, \\
& 2-Z C_{1}^{*}\left(B_{t}\left(C_{n}, v\right)\right)=4 t n+36 t-50 \\
& 3-Z C_{1}^{*}\left(B_{t}\left(K_{n}, v\right)\right)=2 n t(2 n-1)-2 n(2 n+1)+2 t-4 .
\end{aligned}
$$

Proof. 1) The bridge molecular graph $\mathrm{B}_{\mathrm{t}}\left(\mathrm{P}_{\mathrm{n}} ; \mathrm{v}\right)$ has tn vertices. The partition of vertex set based on the degree and the connection number of the vertices is discussed in Table 5. By using Table 5 we can obtain the result

$$
Z C_{1}^{*}\left(B_{t}\left(P_{n}, v\right)\right)=\sum_{v \in V\left(B_{t}\left(P_{n}, v\right)\right)} d(v) \tau_{v}=t \cdot 1 \cdot 1+t \cdot 2 \cdot 1+(t n-4 t+2) \cdot 2 \cdot 2+t \cdot 2 \cdot 3+2 \cdot 3 \cdot 4+(t-4) \cdot 3 \cdot 5=4 t n+8 t-28
$$

2) The bridge molecular graph $\mathrm{B}_{\mathrm{t}}\left(\mathrm{C}_{\mathrm{n}} ; \mathrm{v}\right)$ has th vertices. The partition of vertex set based on the degree and the connection number of the vertices is discussed in Table 6. By using Table 6 we can obtain the result

$$
\begin{aligned}
& Z C_{1}^{*}\left(B_{t}\left(C_{n}, v\right)\right)=\sum_{v \in V\left(B_{t}\left(C_{n}, v\right)\right)} d(v) \tau_{v}=(t n-3 t) \cdot 2 \cdot 2+4 \cdot 2 \cdot 3 \\
& +2(t-2) \cdot 2 \cdot 4+2 \cdot 3 \cdot 5+2 \cdot 4 \cdot 7+(t-4) \cdot 4 \cdot 8=4 t n+36 t-50
\end{aligned}
$$

3) The bridge molecular graph $\mathrm{B}_{\mathrm{t}}\left(\mathrm{K}_{\mathrm{n}}\right.$; v) has tn vertices, and the partition of vertex set based on the degree and the connection number of the vertices is discussed in Table 7. By using Table 7 we can obtain the result

$$
\begin{aligned}
& Z C_{1}^{*}\left(B_{t}\left(K_{n}, v\right)\right)=\sum_{v \in V\left(B_{t}\left(K_{n}, v\right)\right)} d(v) \tau_{v}=2(n-1) \cdot(n-1) \cdot 1+(t-2)(n-1) \cdot(n-1) \cdot 2+2 \cdot n \cdot n \\
& +2 \cdot(n+1) \cdot(2 n-1)+(t-4) \cdot(n+1) \cdot 2 n=2 n t(2 n-1)-2 n(2 n+1)+2 t-4
\end{aligned}
$$

Table 5. Vertex set the partition of bridge molecular graph $\mathrm{B}_{\mathrm{t}}\left(\mathrm{P}_{\mathrm{n}} ; \mathrm{v}\right)$ based on the degree and the connection number of the vertices.

\begin{tabular}{ccc}
\hline $\boldsymbol{d}(\boldsymbol{v})$ & $\boldsymbol{\tau}_{\boldsymbol{v}}$ & Number of vertices \\
\hline 1 & 1 & $t$ \\
\hline 2 & 1 & $t$ \\
\hline 2 & 2 & $t n-4 t+2$ \\
\hline 2 & 3 & 2 \\
\hline 3 & 4 & $t-4$ \\
\hline 3 & 5 &
\end{tabular}

\subsection{Modified the first Zagreb connection index of dendrimer structures.}

Dendrimers are highly ordered branched macromolecules that have attracted much theoretical and experimental attention. This section computes the closed results of the modified first Zagreb index of certain dendrimer structures.

Table 6. Vertex set the partition of bridge molecular graph $\mathrm{B}_{\mathrm{t}}\left(\mathrm{C}_{\mathrm{n}} ; \mathrm{v}\right)$ based on the degree and the connection number of the vertices.

\begin{tabular}{ccc}
\hline $\boldsymbol{d}(\boldsymbol{v})$ & $\boldsymbol{\tau}_{\boldsymbol{v}}$ & Number of vertices \\
\hline 2 & 2 & $t n-3 t$ \\
\hline 2 & 3 & 4 \\
\hline 2 & 4 & $2(t-2)$ \\
\hline 3 & 5 & 2 \\
\hline 4 & 7 & 2 \\
\hline 4 & 8 & $t-4$ \\
\hline
\end{tabular}


The first class of these macromolecules is named $\mathrm{NS}_{1}[\mathrm{n}]$, where $\mathrm{n}$ is the defining parameter. The molecular graph of the $\mathrm{NS}_{1}[\mathrm{n}]$ for $\mathrm{n}=2$ is shown in Fig. 6 . The structure of $\mathrm{NS}_{1}[\mathrm{n}]$ contains $120 \times 2^{\mathrm{n}}-108$ vertices and $140 \times 2^{\mathrm{n}}-127$ edges. Based on the degree and the connection number of the vertices, we partitioned the vertex set. Table 8 illustrate this partition.

Table 7. Vertex set the partition of bridge molecular graph $\mathrm{B}_{\mathrm{t}}\left(\mathrm{K}_{\mathrm{n}} ; \mathrm{v}\right)$ based on the degree and the connection number of the vertices.

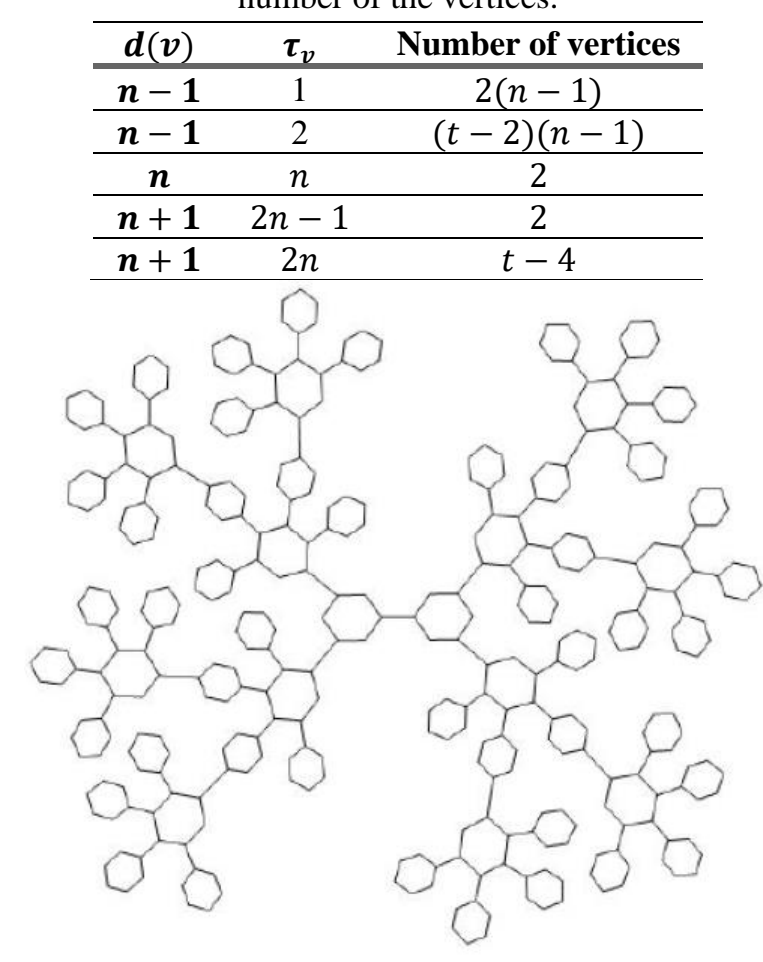

Figure 6. The first type of nanostar dendrimer $\mathrm{NS}_{1}[\mathrm{n}]$.

Theorem 3.5.1. Consider the molecular graph $\mathrm{NS}_{1}[\mathrm{n}]$, where $\mathrm{n} \geq 1$, of the nanostar dendrimer, then its modified first Zagreb connection number is equal to:

$$
\mathrm{ZC}_{1}\left(\mathrm{NS}_{1}[\mathrm{n}]\right)=992 \times 2^{\mathrm{n}}-920 \text {. }
$$

Table 8. Vertex set the partition of $\mathrm{NS}_{1}[\mathrm{n}]$ dendrimer structure based on the degree and the connection number of the vertices.

\begin{tabular}{ccc}
\hline $\boldsymbol{d}(\boldsymbol{v})$ & $\boldsymbol{\tau}_{\boldsymbol{v}}$ & Number of vertices \\
\hline 2 & 2 & $36 \times 2^{n}-24$ \\
\hline 2 & 3 & $40 \times 2^{n}-48$ \\
\hline 2 & 4 & $4 \times 2^{n}+2$ \\
\hline 3 & 4 & $20 \times 2^{n}-18$ \\
\hline 3 & 5 & $8 \times 2^{n}-8$ \\
\hline 3 & 6 & $12 \times 2^{n}-12$ \\
\hline
\end{tabular}

Proof. The molecular graph of $\mathrm{NS}_{1}[\mathrm{n}]$ contains $120 \times 2^{\mathrm{n}}-108$ vertices, and these vertices are of degree two and three. From Fig. 6, we can notice that $27 \times 2^{n}-{ }^{1}-12$ vertices are of degree two and $12 \times 2^{\mathrm{n}-1}-8$ vertices are of degree three. In Table 8 , we partitioned these vertices based on their connection number and used it to obtain the result

$$
\begin{aligned}
& Z C_{1}^{*}\left(N S_{1}[n]\right)=\sum_{v \in V\left(N S_{1}[n]\right)} d(v) \tau_{v}=\left(36 \cdot 2^{n}-24\right) \cdot 2 \cdot 2+\left(40 \cdot 2^{n}-48\right) \cdot 2 \cdot 3 \\
& +\left(4 \cdot 2^{n}+2\right) \cdot 2 \cdot 4+\left(20 \cdot 2^{n}-18\right) \cdot 3 \cdot 4+\left(8 \cdot 2^{n}-8\right) \cdot 3 \cdot 5+\left(12 \cdot 2^{n}-12\right) \cdot 3 \cdot 6=992 \cdot 2^{n}-920
\end{aligned}
$$


We consider the second type of nanostar dendrimers, $\mathrm{NS}_{2}[\mathrm{n}]$, where $\mathrm{n}$ is the defining parameter. Fig. 7 illustrates this type of nanostar dendrimer. This nanostar dendrimer contains $18 \times 2^{\mathrm{n}}-12$ vertices. The vertex partition based on the degree and the connection number of the vertices is shown in the following table.

Table 9. Vertex set the partition of $\mathrm{NS}_{2}[\mathrm{n}]$ dendrimer structure based on the degree and the connection number of the vertices.

\begin{tabular}{ccc}
\hline $\boldsymbol{d}(\boldsymbol{v})$ & $\boldsymbol{\tau}_{\boldsymbol{v}}$ & Number of vertices \\
\hline 2 & 2 & $9 \times 2^{n-1}$ \\
\hline 2 & 3 & $6 \times 2^{n-1}$ \\
\hline 2 & 4 & $9 \times 2^{n-1}-6$ \\
\hline 3 & 4 & $6 \times 2^{n}-6$ \\
\hline
\end{tabular}

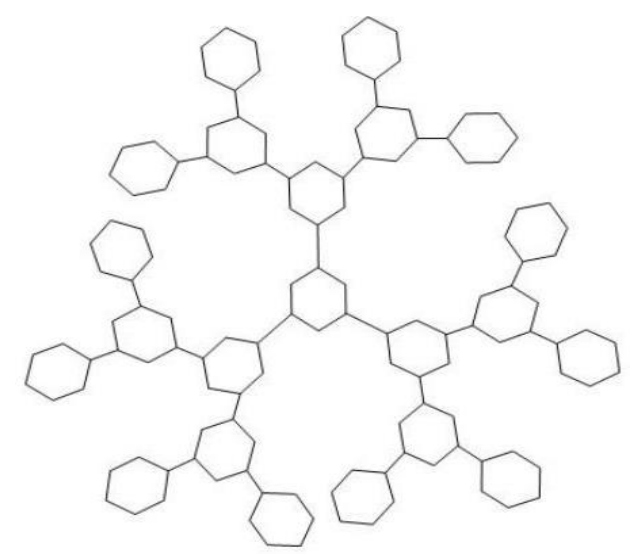

Figure 7. The second type of nanostar dendrimer $\mathrm{NS}_{2}[\mathrm{n}]$.

Theorem 3.5.2. The Modified first Zagreb connection number index of the molecular graph of $\mathrm{NS}_{2}[\mathrm{n}]$, where $\mathrm{n} \geq 1$, is equal to:

$$
\mathrm{ZC}_{1}\left(\mathrm{NS}_{2}[\mathrm{n}]\right)=144 \times 2^{\mathrm{n}}-120
$$

Proof. There are $18 \times 2^{\mathrm{n}}-12$ vertices in the structure of $\mathrm{NS}_{2}[\mathrm{n}]$ and the degree of these vertices are two and three. We partitioned the vertex set of $\mathrm{NS}_{2}[\mathrm{n}]$ based on the vertices' degree and connection number. Table 9 shows this partition. We apply the definition of the modified first Zagreb connection number index to Table 9 to obtain the required result.

$$
Z C_{1}^{*}\left(N S_{2}[n]\right)=\sum_{v \in V\left(N S_{2}[n]\right)} d(v) \tau_{v}=9 \cdot 2^{n-1} \cdot 2 \cdot 2+6 \cdot 2^{n-1} \cdot 2 \cdot 3+\left(9 \cdot 2^{n-1}-6\right) \cdot 2 \cdot 4+\left(6 \cdot 2^{n}-6\right) \cdot 3 \cdot 4=144 \cdot 2^{n}-120
$$

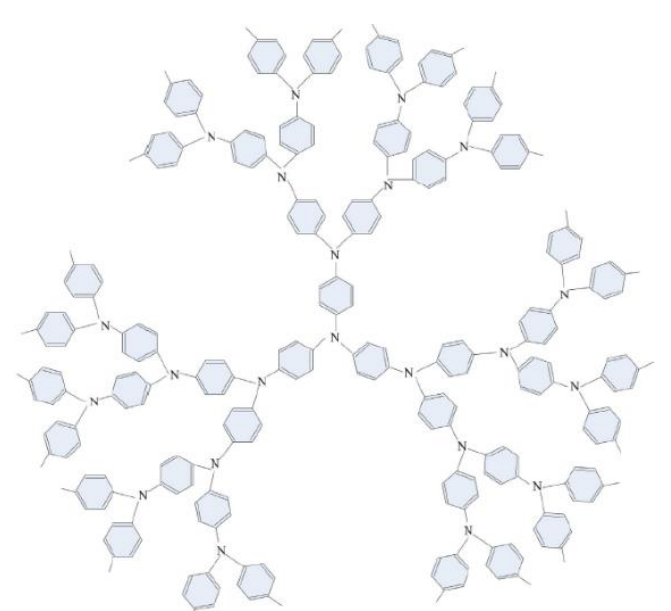

Figure 8. The molecular graph of the star dendrimer $\mathrm{D}_{3}[\mathrm{n}]$. 
The third type of star dendrimer is denoted as $D_{3}[n]$, where $n$ represents the $n^{\text {th }}$ growth of star dendrimer and $\mathrm{n}$ 0. The molecular graph of the star dendrimer $\mathrm{D}_{3}[\mathrm{n}]$ is shown in Fig. 8 . Theorem 6.3. Consider the molecular graph $\mathrm{D}_{3}[\mathrm{n}]$, where $\mathrm{n} 1$, of the star dendrimer, then its modified first Zagreb connection number is

$$
\mathrm{ZC}_{1}\left(\mathrm{D}_{3}[\mathrm{n}]\right)=306 \times 2^{\mathrm{n}}-{ }_{-1}-180
$$

Proof. The molecular graph of $\mathrm{D}_{3}[\mathrm{n}]$ chain contains $39 \times 2^{\mathrm{n}}-{ }^{1}-20$ vertices. From Fig. 8, one can notice that there are $272^{\mathrm{n}-1}-12$ vertices of degree two and $12 \times 2^{\mathrm{n}-1}-8$ vertices of degree three. With the help of Table 10, we can easily compute the modified first Zagreb connection index of the $\mathrm{D}_{3}[\mathrm{n}]$ as follows

$$
Z C_{1}^{*}\left(D_{3}[n]\right)=\sum_{v \in V\left(D_{3}[n]\right)} d(v) \tau_{v}=9 \cdot 2^{n-1} \cdot 2 \cdot 2+18 \cdot 2 n-1-12 \cdot 2 \cdot 3+9 \cdot 2^{n-1}-6 \cdot 3 \cdot 4+3 \cdot 2^{n-1}-2 \cdot 3 \cdot 6
$$

After a simple calculation, we have the result $Z C_{1}^{*}\left(D_{3}[n]\right)=306 \cdot 2^{n-1}-180$.

Table 10. Vertex set the partition of the star dendrimer's molecular graph based on the degree and the connection number of the vertices.

\begin{tabular}{ccc}
\hline $\boldsymbol{d}(\boldsymbol{v})$ & $\boldsymbol{\tau}_{\boldsymbol{v}}$ & Number of vertices \\
\hline 2 & 2 & $9 \times 2^{n-1}$ \\
\hline 2 & 3 & $18 \times 2^{n-1}-12$ \\
\hline 3 & 4 & $9 \times 2^{n-1}-6$ \\
\hline 3 & 6 & $3 \times 2^{n}-2$ \\
\hline
\end{tabular}

3.6. Modified first Zagreb connection number of Benzenoid series class.

In this section, we compute the modified first Zagreb connection number of two Benzenoid series classes, namely the Circumcoronene series of Benzenoid $\mathrm{H}_{\mathrm{t}}$. The molecular graph of $\mathrm{H}_{\mathrm{t}}$ is shown in Fig. 9.

Table 11. Vertex set the partition of the molecular graph of the Circumcoronene Benzenoid series $\mathrm{H}_{\mathrm{t}}$ based on the degree and the connection number of the vertices.

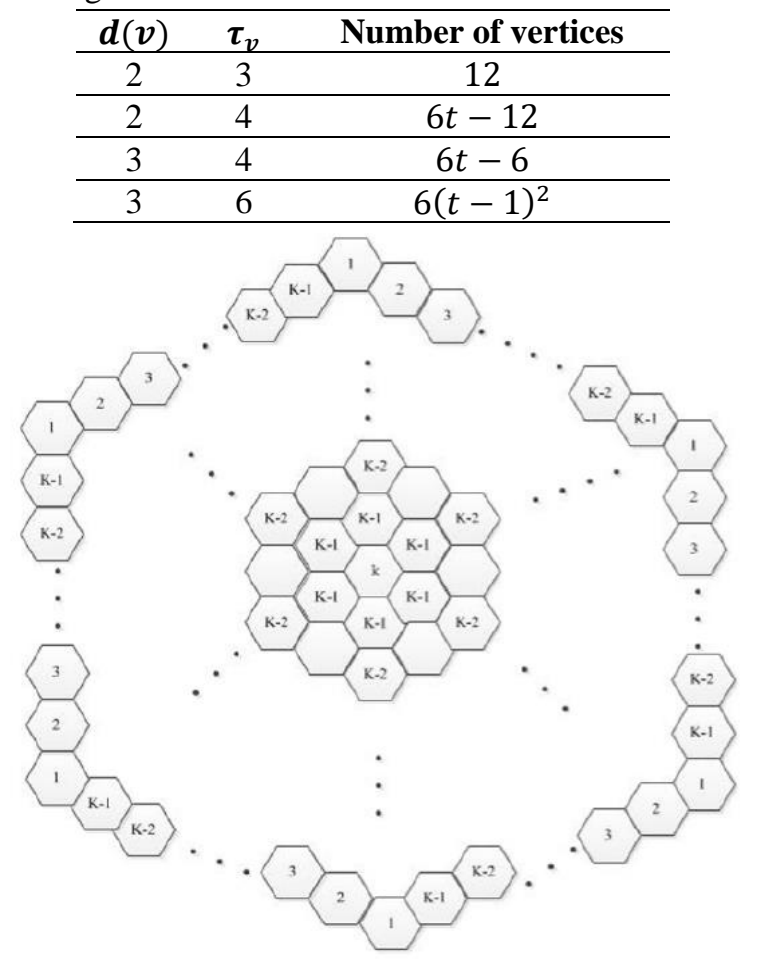

Figure 9. The molecular graph of the Circumcoronene series of Benzenoid $\mathrm{H}_{\mathrm{t}}$. 
Theorem 3.6.1. The modified first Zagreb connection index of the molecular graph of Circumcoronene series of Benzenoid $\mathrm{H}_{\mathrm{t}}, \mathrm{t} \geq 1$, is given as:

$$
\mathrm{ZC}_{1}\left(\mathrm{H}_{\mathrm{t}}\right)=108 \times \mathrm{t}^{2}-96 \mathrm{t}+12 \text {. }
$$

Proof. There are total of $6 \mathrm{t}^{2}$ vertices in the molecular graph of Circumcoronene Benzenoid series $\mathrm{H}_{\mathrm{t}}$. There are only two types of vertices in this structure with respect to degrees, vertices with degree two and vertices with degree 3 . There are $6 t$ vertices of degree two and $6 t^{2}-6 t$ vertices of degree three. Table 11 partitioned the vertex set $\mathrm{V}\left(\mathrm{H}_{\mathrm{t}}\right)$ based on the degree and the connection number of the vertices. Since

$$
Z C_{1}^{*}\left(H_{t}\right)=\sum_{v \in V\left(H_{t}\right)} d(v) \tau_{v}=12 \cdot 2 \cdot 3+6(t-2) \cdot 2 \cdot 4+6(t-1) \cdot 3 \cdot 4+6(t-1)^{2} \cdot 3 \cdot 6=108 \cdot t^{2}-96 t+12 .
$$

\section{Conclusions}

This paper computes the modified first Zagreb connection index for several vastly studied chemical graphs. Modified first Zagreb index depends on the degrees and connection numbers of the vertices of the graph. Our computed results may have an important role in the study of $\pi$-electron energy of dendrimers. Using our computed results, the future targets can be obtained by the collaboration of mathematicians and pharmacists.

\section{Funding}

This research received no external funding.

\section{Acknowledgments}

All the authors are thankful to their respective institutions.

\section{Conflicts of Interest}

The authors declare no conflict of interest.

\section{References}

1. Gutman, I.; Polansky, O.E. Mathematical concepts in organic chemistry. Springer: Berlin, 1986; https://doi.org/10.1007/978-3-642-70982-1.

2. Trinajstic, N. Chemical Graph Theory. $2^{\text {nd }}$ ed.; CRC Press, Boca Raton: Florida, 1992.

3. Furtula, B.; Gutman, I. A forgotten topological index. J. Math. Chem. 2015, 53, 1184-1190, https://doi.org/10.1007/s10910-015-0480-z.

4. Gao, W.; Iqbal, Z.; Ishaq, M.; Aslam, A.; Aamir, M.; Binyamin, M.A. Bounds on Topological Descriptors of the Corona Product of F-Sum of Connected Graphs. IEEE Access 2019, 7, 26788-26796, https://doi.org//10.1109/ACCESS.2019.2900061.

5. Gao, W.; Iqbal, Z.; Ishaq, M.; Sarfraz, R.; Aamir, M.; Aslam, A. On eccentricity-based topological indices study of a class of porphyrin-cored dendrimers. Biomolecules 2018, 8, https://doi.org/10.3390/biom8030071.

6. Gao, Y.; Zhu, E.; Shao, Z.; Gutman, I.; Klobučar, A. Total domination and open packing in some chemical graphs. J. Math. Chem. 2018, 56, 1481-1492, https://doi.org/10.1007/s10910-018-0877-6.

7. Yang, H.; Imran, M.; Akhter, S.; Iqbal, Z.; Siddiqui, M.K. On Distance-Based Topological Descriptors of Subdivision Vertex-Edge Join of Three Graphs. IEEE Access 2019, 7, 143381-143391, https://doi.org/10.1109/ACCESS.2019.2944860.

8. Iqbal, Z.; Aslam, A.; Ishaq, M.; Gao, W. The Edge Versions of Degree-Based Topological Descriptors of Dendrimers. J. Cluster Sci. 2020, 31, 445-452, https://doi.org/10.1007/s10876-019-01658-w.

9. Zheng, J.; Iqbal, Z.; Fahad, A.; Zafar, A.; Aslam, A.; Qureshi, M.I.; Irfan, R. Some eccentricity-based topological indices and polynomials of poly (EThyleneAmidoAmine)(PETAA) dendrimers. Processes 2019, 7, https://doi.org/10.3390/pr7070433. 
10. Gao, W.; Akhter, S.; Iqbal, Z.; Qasim, M.; Aslam, A. The Topological Aspects of Phthalocyanines and $\begin{array}{llllll}\text { Porphyrins } \quad \text { Dendrimers. } & \text { IEEE }\end{array}$ https://doi.org/10.1109/ACCESS.2020.3023658.

11. Zahid, I.; Muhammad, I.; Adnan, A.; Wei, G. On eccentricity-based topological descriptors of water-soluble dendrimers. Zeitschrift für Naturforschung C 2019, 74, 25-33, https://doi.org/10.1515/znc-2018-0123.

12. Luo, L.; Dehgardi, N.; Fahad, A. Lower Bounds on the Entire Zagreb Indices of Trees. Discrete Dynamics in Nature and Society 2020, 2020, https://doi.org/10.1155/2020/8616725.

13. Zhang, X.; Raza, A.; Fahad, A.; Jamil, M.K.; Chaudhry, M.A.; Iqbal, Z. On Face Index of Silicon Carbides. Discrete Dynamics in Nature and Society 2020, 2020, https://doi.org/10.1155/2020/6048438.

14. Ye, A.; Javed, A.; Jamil, M.K.; Abdul Sattar, K.; Aslam, A.; Iqbal, Z.; Fahad, A. On Computation of Face Index of Certain Nanotubes. Discrete Dynamics in Nature and Society 2020, 2020, https://doi.org/10.1155/2020/3468426.

15. Imran, M.; Nawaz, T.; Malik, M.A.; Jamil, M.K.; Hayat, S. On topological properties of boron and boron- $\alpha$ nanotubes. Mathematical methods in applied sciences 2020, 1-15.

16. Zhao, D.; Iqbal, Z.; Irfan, R.; Chaudhry, M.A.; Ishaq, M.; Jamil, M.K.; Fahad, A. Comparison of Irregularity Indices of Several Dendrimers Structures. Processes 2019, 7, https://doi.org/10.3390/pr7100662.

17. Wei, C.-C.; Ali, H.; Binyamin, M.A.; Naeem, M.N.; Liu, J.-B. Computing Degree Based Topological Properties of Third Type of Hex-Derived Networks. Mathematics 2019, 7, https://doi.org/10.3390/math7040368 .

18. Ali, H.; Binyamin, M.A.; Shafiq, M.K.; Gao, W. On the Degree-Based Topological Indices of Some Derived Networks. Mathematics 2019, 7, https://doi.org/10.3390/math7070612.

19. Iqbal, Z.; Aslam, A.; Ishaq, M.; Aamir, M. Characteristic study of irregularity measures of some nanotubes. Canadian Journal of Physics 2019, 97, 1125-1132, https://doi.org/10.1139/cjp-2018-0619.

20. Gao, W.; Aamir, M.; Iqbal, Z.; Ishaq, M.; Aslam, A. On Irregularity Measures of Some Dendrimers Structures. Mathematics 2019, 7, https://doi.org/10.3390/math7030271.

21. Ye, A.; Qureshi, M.I.; Fahad, A.; Aslam, A.; Jamil, M.K.; Zafar, A.; Irfan, R. Zagreb Connection Number Index of Nanotubes and Regular Hexagonal Lattice. Open Chemistry 2019, 17, 75-80, https://doi.org/10.1515/chem-2019-0007.

22. Jalil, A.; Javed, A.; Jamil, M.K.; Alaeiyan, M.; Farahani, M.R. Topological properties of four types of porphyrin dendrimers. Proyecciones Journal of Mathematics 2020, 39, 979-993, https://doi.org/10.22199/issn.0717-6279-2020-04-0061.

23. Alfuraidan, M.R.; Imran, M.; Jamil, M.K.; Vetrik, T. General multiplicative Zagreb indices of graphs with indices. IEEE Access 2020, 118725-118731, https://doi.org/10.1109/ACCESS.2020.3005040.

24. Mojdeh, D.A.; Habibi, M.; Badakhshian, L.; Rao, Y. Zagreb indices of trees, unicyclic and bicyclic graphs with given (total) domination. IEEE Access 2019, 7, 94143-94149, https://doi.org/10.1109/ACCESS.2019.2927288.

25. Liu, J.B.; Javaid, M.; Awais, H.M. Computing Zagreb indices of the subdivision-related generalized $\begin{array}{lllll}\text { operations of } & \text { graphs, }\end{array}$ https://doi.org/10.1109/ACCESS.2019.2932002.

26. Bashir, Y.; Aslam, A.; Kamran, M.; Qureshi, M.I.; Janagir, A.; Rafiq, M.; Bibi, N.; Muhammad, N. On Forgotten Topological indices of some Dendrimers Structure. Molecules 2017, 22, https://doi.org/10.3390/molecules22060867.

27. Munir, M.; Nazeer, W.; Kang, S.M.; Qureshi, M.I.; Nizami, A.R.; Kwun, Y.C. Some Invariants of Jahangir Graphs. Symmetry 2017, 9, https://doi.org/10.3390/sym9010017.

28. Gutman, I.; Trinajstic, N. Graph theory and molecular orbitals. Total $\pi$-electron energy of alternant hydrocarbons. Chem. phys. Lett. 1972, 17, 535-538, https://doi.org/10.1016/0009-2614(72)85099-1.

29. Ali, A.; Trinajstić, N. A novel/old modification of the first Zagreb index. Molecular Informatics 2018, 37 , 6-7, https://doi.org/10.1002/minf.201800008.

30. Goodman, J.E.; Rourke, J.O. Handbook of Discrete and Computational Geometry. CRC Press LLC 1997; pp. 225-242. 